\title{
ACL update: objective measures on knee instability: an introduction
}

\author{
Volker Musahl $^{1} \cdot$ Anil Ranawat $^{2}$
}

Published online: 12 March 2016

(C) Springer Science+Business Media New York 2016

Keywords ACL $\cdot$ Knee stability $\cdot$ Pivot shift $\cdot$ Knee

Successful anterior cruciate ligament (ACL) reconstruction is defined by the absence of osteoarthritis (OA) and complete return of knee function. The difficulty with measuring these outcomes is that OA has to be measured at up to 10 years post-surgery and testing of knee function requires complicated and not readily available tools, such as dynamic stereo radiography (DSX). However, there are several new measures of dynamic and static knee instability that can be used by clinicians and scientists to measure outcomes.

Knee instability is the one surrogate outcome correlating with patient satisfaction following ACL reconstruction. In other words, successful ACL reconstruction is the lack of persistent knee instability, particularly rotatory knee instability. In this theme issue of Current Reviews in Musculoskeletal Medicine, we review basic principles of knee biomechanics

and illustrate how to measure knee instability using imaging tools including magnet resonance imaging (MRI) and stress radiography. Several tools have been developed recently to assess rotatory knee laxity in three general categories: (1) Static rotatory laxity, which can give pre- and post-operative insights on knee function and individual laxity profiles; (2) Dynamic rotatory laxity, which is accomplished by video motion analysis or DSX; (3) Pivot shift, which is quantifiable utilizing inertial sensors, electromagnetic tracking, or image analysis.

Technological advancements over the last few years have helped in the development of many new devices that will aid in assessing clinical outcomes after ACL reconstruction. Innovatively, the noninvasiveness of the objective measurement devices allows for use in the clinic setting on conscious patients. Future research will further improve and develop new devices as well as establish better treatment algorithms for ACL reconstruction with the ultimate goal of improving outcomes for patients.

This article is part of the Topical Collection on ACL Update: Objective Measures on Knee Instability

Volker Musahl

musahlv@upmc.edu

Anil Ranawat

ranawatanil@hss.edu

1 University of Pittsburgh, UPMC Center for Sports Medicine, 3200 South Water Street, Pittsburgh, PA 15203, USA

2 Hospital for Special Surgery, 535 E. 70th, 6th floor, New York, NY 10021, USA 